\title{
INVENTÁRIO DA ARBORIZAÇÃO URBANA NO MUNICÍPIO DE ARAÇOIABA DA SERRA, SP
}

\author{
Thaís Graciano-Silva ${ }^{1}$, Eliana Cardoso-Leite ${ }^{2}$, Kelly Cristina Tonello ${ }^{3}$
}

\section{RESUMO}

Em contextos urbanos, nos quais os elementos naturais são cada vez mais suprimidos para que se dê avanço ao desenvolvimento das cidades, parques, praças e ruas bem arborizadas apresentam uma perspectiva de fuga do cotidiano de modo a proporcionar contato com o meio ambiente, amenizando impactos negativos decorrentes da poluição visual, sonora, atmosférica e hídrica. O presente trabalho teve como objetivo a realização de um inventário arbóreo urbano no Município de Araçoiaba da Serra, realizado em 67 ruas pertencentes às zonas central e residencial mista. A coleta de dados quali-quantitativos abrangeu 1542 indivíduos, distribuídos em 121 espécies, sendo mais frequentemente encontradas as espécies Ficus benjamina, Caesalpinia pluviosa var. peltophoroides e Handroanthus chrysotrichus. Foram analisadas, também, a fitossanidade, a situação do sistema radicular, o diâmetro a altura do peito, a altura da primeira bifurcação, a altura total, a posição da fiação em relação a copa e o diâmetro da copa; sendo encontrados, em geral, poucos problemas com a arborização local, muito disto decorrente do fato do município ainda ser pouco desenvolvido com relação às estruturas físicas que costumam estar presentes em centros urbanos.

Palavras-chave: Levantamento Florístico; Planejamento Urbano; Vegetação Urbana.

Recebido em 22.05.2014 e aceito em 25.05.2015

1 Bióloga, UFSCar campus Sorocaba, PPGSGA- Programa de Pós Graduação em Sustentabilidade na Gestão Ambiental. thagraciano@gmail.com

2 Bióloga, UFSCar campus Sorocaba, Departamento de Ciências Ambientais, Sorocaba, SP, Brasil, PPGSGA- Programa de Pós Graduação em Sustentabilidade na Gestão Ambiental. eliana.leite@ufscar.br

3 Engenheira Florestal, UFSCar campus Sorocaba, Departamento de Ciências Ambientais, Sorocaba, SP, Brasil. PPGSGAPrograma de Pós Graduação em Sustentabilidade na Gestão Ambiental. kellytonello@ufscar.br 
INVENTORY OF THE URBAN FORESTRY IN ARAÇOIABA DA SERRA, SP.

\begin{abstract}
In urban contexts, in which natural elements are getting increasingly suppressed so that the cities can develop themselves, parks, squares and wooded streets presents a prospect of escape from the everyday in order to provide contact with the environment, assuaging negative impacts accrued from visual, noise, air and water pollution. The present study aimed to accomplish an urban arboreal inventory in the county of Araçoiaba da Serra, conducted by obtaining qualitative and quantitative data collected from arboreal and shrubby individuals located in the streets of the city compassing 1542 inventoried individuals, distributed in 121 species among which the most frequently found species are Ficus benjamina, Caesalpinia pluviosa var. peltophoroides and Handroanthus chrysotrichus. The phyto-sanity, root system situation, diameter at breast height, height of the first fork, total height, the relationship between electrical wiring and the canopy and crown diameter were also analyzed; finding, in general, few problems with the local afforestation, much of it due to the fact that the city is still a little underdeveloped in terms of physical structures that are usually present in urban centers.
\end{abstract}

Keywords: Floristic Survey; Urban Planning; Urban Vegetation.

\title{
INTRODUÇÃO
}

No século XIX, a presença de vegetação nas cidades ia de encontro com a fase desenvolvimentista do período, sendo então, valorizados os espaços urbanos que não contemplavam o verde em sua paisagem (SHAMS et al., 2009). Já no início do século XX surgiram, em números significativos, os jardins, as praças e os parques arborizados, como resultado da percepção da população acerca das áreas verdes como componentes necessários aos espaços urbanos, bem como da perda da qualidade de vida diante do distanciamento dos ambientes naturais (GOMES; SOARES, 2003). Desde então, percebeuse que a manutenção da vida nas grandes cidades está intimamente relacionada com a existência de árvores, seja em conjuntos formando fragmentos florestais ou isoladamente 
como na arborização urbana. Estas interferem diretamente na qualidade de vida da população local por meio de suas funções ecológicas, sociais, estéticas e educativas, além de amenizar as percepções negativas geradas pela urbanização (MATIAS; CAPORUSSO, 2008).

O aprofundamento dos estudos relacionados à arborização urbana faz-se cada vez mais necessário, uma vez que a falta do planejamento urbano adequado acaba culminando em iniciativas particulares pontuais e desprovidas de conhecimento técnico, acarretando, muitas vezes, a perda da eficácia do conforto físico e psíquico que a presença de árvores pode gerar no contexto urbano (ALMEIDA; RONDON NETO, 2010). A maioria das cidades brasileiras teve sua arborização implantada desordenadamente sem que houvesse qualquer tipo de planejamento, o que a configurou como um grande gerador de conflitos na atualidade, assim, obter uma avaliação do estado recente dos indivíduos, através de seu inventário, pode favorecer a elaboração de um planejamento mais eficiente tanto para as áreas com a arborização já implementadas como em locais em que ainda se pretende arborizar (SILVA et al., 2007).

O inventário visa obter informações a respeito da arborização presente no ambiente urbano, de forma que seja possível conhecer as espécies presentes nestes locais e seu estado de saúde, bem como estudar como se distribuem no território. Sua realização proporciona, também, a detecção de possíveis necessidades de manejo e a localização de novas áreas para plantio (SILVA et al., 2007).

Diante de tal importância, o presente trabalho teve como objetivo a realização de um inventário quali-quantitativo da vegetação arbórea e arbustiva presente nas zonas central e residencial mista do município de Araçoiaba da Serra, a fim de diagnosticar o estado fitossanitário e dendrológico dos indivíduos, bem como apontar os possíveis conflitos existentes entre a arborização local e as estruturas urbanas.

\section{MATERIAIS E MÉTODOS}

\section{Área de Estudo}

O Município de Araçoiaba da Serra localiza-se no interior do Estado de São Paulo (Figura 1), a $115 \mathrm{Km}$ da capital, abragendo uma área territorial de $255 \mathrm{Km}^{2}$, possui uma população de 27.299 habitantes (IBGE, 2013). Localizada entre dois biomas brasileiros, (Mata Atlântica e Cerrado) conta com as formações vegetacionais de Cerrado, Floresta 
Estacional Semidecidual e Floresta Ombrófila Densa (IBGE, 2012). O clima da região é do tipo Cfa (clima subtropical quente e úmido), segundo a classificação de Köpen, com temperatura média anual de $21^{\circ} \mathrm{C}$ e pluviosidade média anual de $1400 \mathrm{~mm}$ (ARAÇOIABA DA SERRA, 2010).

Figura 1. Localização do Município de Araçoiaba da Serra, Estado de São Paulo, Brasil Figure 1. Location of the Municipality of Araçoiaba da Serra, São Paulo, Brazil

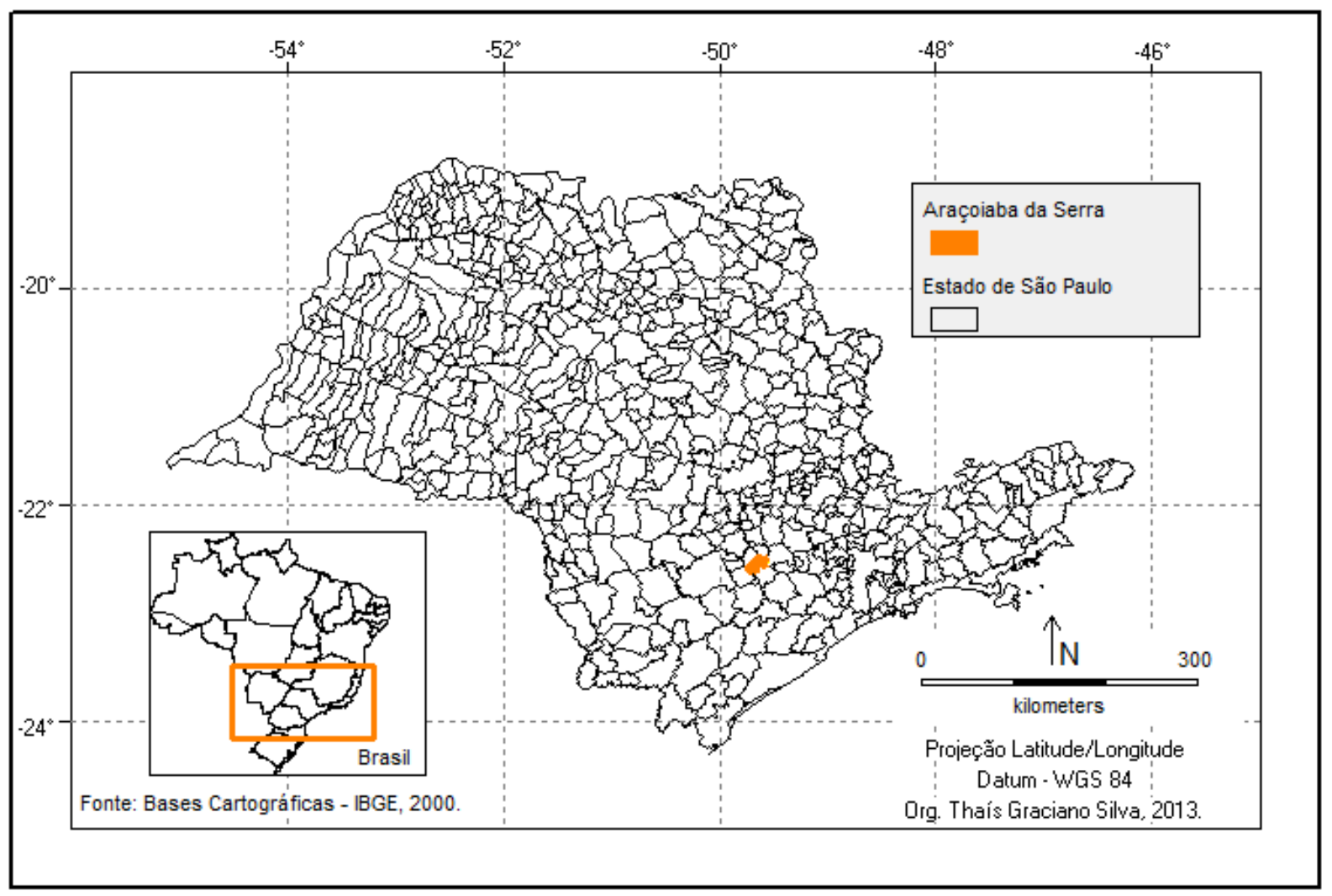

\section{Coleta e análise de dados}

O inventário foi realizado entre abril e junho de 2013, através de amostragem qualiquantitativa realizada em 67 ruas localizadas nas zonas central e residencial mista do município (Figura 2). A escolha da amostra se deu de forma aleatória através de sorteio dentre aquelas pertencentes às duas zonas citadas, sendo consideradas $20 \%$ das 333 ruas que constam oficialmente nas zonas selecionadas. Foram descartadas áreas pertencentes à zona rural, zona de chácaras e zona industrial devido a estas apresentarem, em sua maioria, apenas acessos vicinais sem espaços para passeio público ou arborização; descartou-se também a zona predominantemente residencial uma vez que esta compreende os condomínios e loteamentos fechados, os quais poderiam criar um viés na amostragem 
uma vez que apresentam padrões de arborização pensados por profissionais contratados para manter o paisagismo destes locais.

Figura 2. Delimitação da cidade de Araçoiaba da Serra e localização das 67 ruas abrangidas neste inventário

Figure 2. Delimitation of the city of Araçoiaba da Serra and the location of the 67 streets covered in this inventory

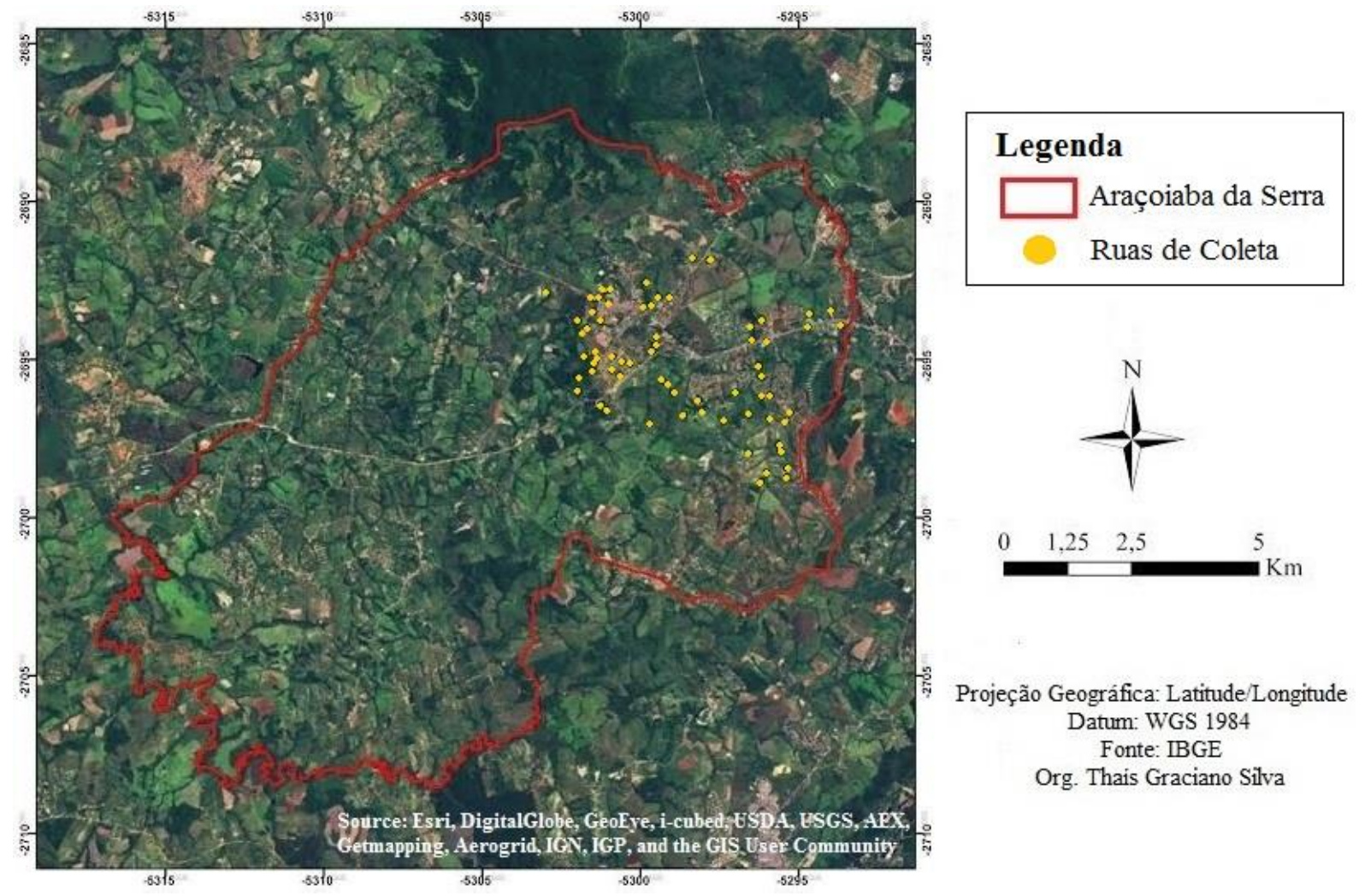

A coleta de dados seguiu a metodologia também utilizada por Silva et al.(2007) sendo inventariados indivíduos arbóreos e arbustivos, com diâmetro a altura do peito maior ou igual a cinco centímetros e altura igual ou superior a um metro; cada indivíduo foi identificado quanto ao seu nome popular, à família e a espécie a que pertence, sendo os demais dados, como situação da raiz diante do passeio, interferência da copa dos indivíduos na fiação elétrica, fitossanidade, altura total e da primeira bifurcação, além do diâmetro a altura do peito e do diâmetro da copa anotados em formulários cujo preenchimento de dados seguiu os seguintes critérios:

- Fitossanidade: "Morto" quando se apresentou sem vida ou com morte iminente; "Ruim" quando apresentou um estágio de declínio (com severos danos físicos e injúrias causadas por pragas e doenças); "Regular" quando se apresentava com vigor médio (presença de pequenos danos físicos, necessidade de poda corretiva ou pequenos sinais de 
pragas e doenças) e "Bom" quando mostrou-se vigoroso (sem sinais de pragas, doenças ou danos mecânicos);

- Influência do sistema radicular no passeio público: "Não aparente", "Aparente no solo", "Trincas no pavimento" e "Rompeu o pavimento";

- Diâmetro a altura do peito: As medidas, realizadas a 1,30m do solo, foram alocadas nas seguintes classes, "De 5 a menores que 35cm", "De 35 a menores de 65cm", "De 65 a $95 \mathrm{~cm}$ " e "Maiores que $95 \mathrm{~cm}$ ";

- Altura da primeira bifurcação: "Menor que 1,80m", "Maior que 1,80m" e "Sem bifurcação";

- Altura total: "Menores que 5m", "De $5 \mathrm{~m}$ a $10 \mathrm{~m}$ " e "Maiores que 10m";

- Relação entre a fiação elétrica e a copa: Avaliou-se o posicionamento da fiação elétrica em relação à copa, podendo a fiação estar "Acima da copa", no "Meio da copa", "Abaixo da copa" e "Inexistente";

- Diâmetro da copa: "Menores que 5m", "De 5 a menor que 10m", "De 10 a menor que $15 \mathrm{~m}$ " e "Maior que $15 \mathrm{~m}$ ".

A identificação das espécies foi feita por meio de registro fotográfico e/ou coleta de material botânico para identificação em laboratório. A confirmação das espécies se deu através da comparação com literatura específica como Lorenzi (2008, 2009a, 2009b, 2010), Lorenzi et al. (2003, 2004), Medeiros (2011) e Ramos et al. (2008), sendo os nomes científicos e as famílias a que cada indivíduo pertence, bem como sua origem, conferidos com auxílio de bancos de dados presentes nos sites Lista de Espécies da Flora do Brasil (2013) e Tropicos (2013). As espécies nativas ameaçadas de extinção foram conferidas na lista de espécies ameaçadas da IUCN (2013). Para analisar a diversidade de espécies encontradas nas zonas estudadas, foi aplicado o cálculo do Índice de Diversidade de Shannon-Wiener (H').

\section{RESULTADOS E DISCUSSÃO}

\section{Composição de espécies da arborização viária}

Foram amostrados 1542 indivíduos de porte arbóreo e arbustivo, dentre estes, 72 indivíduos foram considerados mortos, sendo os demais identificados em 121 espécies (44\% nativas e 55\% exóticas), 98 gêneros e 42 famílias botânicas (Tabela 1). Observa-se a predominância de indivíduos pertencentes as famílias Fabaceae (20\%) e Bignoniaceae 
(16,4\%). As espécies Ficus benjamina (6,54\%), Caesalpinia pluviosa var. peltophoroides $(5,7 \%)$ e Handroanthus chrysotrichus (5,38\%) apresentaram as maiores densidades de indivíduos neste inventário. Com relação à diversidade de espécies, o $\mathrm{H}^{\prime}$ foi estimado em 4,08. Dos 1542 indivíduos amostrados 522 pertencem a espécies nativas, 35 indivíduos a uma espécie subespontânea e 985 indivíduos a espécies exóticas. Dentre as espécies nativas encontradas no inventário, cinco delas são consideradas ameaçadas de extinção, sendo elas, Bauhinia forficata Link (Unha-de-boi), Caesalpinia echinata Lam. (Pau Brasil), Cedrela fissilis Vell. (Cedro), Hymenaea courbaril (Jatobá) e Platypodium elegans Vogel (Amendoim do Campo).

Tabela 1. Lista de espécies inventariadas, a família a qual pertencem, seu nome popular, suas frequências e origem ( $\mathrm{N}=$ nativa / $\mathrm{E}=$ exótica / $\mathrm{SE}=$ subespontânea). ${ }^{1}$ Espécies endêmicas; ${ }^{2}$ Espécies nativas ameaçadas de extinção (Categoria IUCN: $\mathrm{E}=$ ameaçada de extinção / LC= menor preocupação)

Table 1. List of identified species, the family to which they belong, its popular name, their frequencies and origin ( $\mathrm{N}=$ native / $\mathrm{E}=$ exotic / $\mathrm{SE}=$ sub-spontaneous). ${ }^{1}$ Endemic species, ${ }^{2}$ Native species endangered of extinction (IUCN Categories: $\mathrm{E}=$ endangered / LC = Least Concern)

\begin{tabular}{|c|c|c|c|c|c|}
\hline \multirow[b]{2}{*}{ Nome Científico } & \multirow[b]{2}{*}{ Família } & \multirow[b]{2}{*}{ Nome Popular } & \multirow[b]{2}{*}{ Origem } & \multicolumn{2}{|c|}{ Frequência } \\
\hline & & & & Absoluta & Relativa \\
\hline Ficus benjamina L. & Moraceae & Ficus & $\bar{E}$ & 101 & 6,54 \\
\hline $\begin{array}{l}\text { Caesalpinia pluviosa var. } \\
\text { peltophoroides (Benth.) G.P. Lewis }\end{array}$ & Fabaceae & Sibipiruna & N & 88 & 5,7 \\
\hline $\begin{array}{l}\text { Handroanthus chrysotrichus (Mart. ex } \\
\text { DC.) Mattos }\end{array}$ & Bignoniaceae & Ipê Amarelo & N & 83 & 5,38 \\
\hline Tabebuia pentaphylla (L.) Hemsl. & Bignoniaceae & Ipê Rosa & $E$ & 69 & 4,47 \\
\hline Delonix regia (Bojer ex Hook.) Raf. & Fabaceae & Flamboyant & $\mathrm{E}$ & 68 & 4,40 \\
\hline Bauhinia variegata $\mathrm{L}$. & Fabaceae & Pata de Vaca & $E$ & 55 & 3,57 \\
\hline Mangifera indica $\mathrm{L}$. & Anacardiaceae & Mangueira & $E$ & 45 & 2,92 \\
\hline Cupressus Iusitanica Mill. & Cupressaceae & Cedrinho & $E$ & 42 & 2,72 \\
\hline Morus nigra L. & Moraceae & Amoreira & $E$ & 38 & 2,46 \\
\hline Psidium guajava L. & Myrtaceae & Goiabeira & SE & 35 & 2,27 \\
\hline Tibouchina granulosa (Desr.) Cogn. ${ }^{1}$ & Melastomataceae & Quaresmeira & $\mathrm{N}$ & 34 & 2,2 \\
\hline Yucca elephantipes Regel & Asparagaceae & Pata de Elefante & $E$ & 33 & 2,14 \\
\hline Jacaranda mimosifolia D. Don & Bignoniaceae & Jacarandá Mimoso & $E$ & 33 & 2,14 \\
\hline Eugenia uniflora L. & Myrtaceae & Pitangueira & $\mathrm{N}$ & 32 & 2,07 \\
\hline Tabebuia roseoalba (Ridl.) Sandwith & Bignoniaceae & Ipê Branco & $\mathrm{N}$ & 29 & 1,88 \\
\hline $\begin{array}{c}\text { Dypsis lutescens ( } \mathrm{H} \text {. Wendl.) Beentje \& } \\
\text { Dransf. }\end{array}$ & Arecaceae & Palmeira Areca & $E$ & 26 & 1,68 \\
\hline Persea americana Mill. & Lauraceae & Abacateiro & $E$ & 26 & 1,68 \\
\hline Koelreuteria bipinnata Franch. & Sapindaceae & Árvore da China & $E$ & 23 & 1,49 \\
\hline Euphorbia cotinifolia L. & Euphorbiaceae & Leiteiro Vermelho & $E$ & 22 & 1,42 \\
\hline Peltophorum dubium (Spreng.) Taub. & Fabaceae & Canafístula & $\mathrm{N}$ & 20 & 1,3 \\
\hline Lagerstroemia indica L. & Lythraceae & Resedá & $E$ & 20 & 1,3 \\
\hline Pinus elliottii Engelm. & Pinaceae & Pinheiro & $E$ & 20 & 1,3 \\
\hline
\end{tabular}




\begin{tabular}{|c|c|c|c|c|c|}
\hline Duranta erecta L. & Verbenaceae & Pingo-de-ouro & $E$ & 20 & 1,3 \\
\hline $\begin{array}{c}\text { Syagrus romanzoffiana (Cham.) } \\
\text { Glassman }\end{array}$ & Arecaceae & Jerivá & $\mathrm{N}$ & 19 & 1,23 \\
\hline Schinus terebinthifolia Raddi & Anacardiaceae & Aroeira Pimenteira & $\mathrm{N}$ & 18 & 1,17 \\
\hline Cedrela fissilis Vell. ${ }^{2} \mathrm{E}$ & Meliaceae & Cedro & $\mathrm{N}$ & 18 & 1,17 \\
\hline Spathodea campanulata P. Beauv. & Bignoniaceae & Espatódea & $E$ & 16 & 1,04 \\
\hline Syagrus picrophylla Barb. Rodr. ${ }^{1}$ & Arecaceae & Licuri & $\mathrm{N}$ & 15 & 0,97 \\
\hline Terminalia catappa L. & Combretaceae & Chapéu de Praia & $E$ & 15 & 0,97 \\
\hline Bougainvillea sp. & Nyctaginaceae & Primavera & $\mathrm{N}$ & 15 & 0,97 \\
\hline Chorisia speciosa A. St.-Hil. & Malvaceae & Paineira & $\mathrm{N}$ & 13 & 0,84 \\
\hline Cordia superba Cham. ${ }^{1}$ & Boraginaceae & Babosa Branca & $\mathrm{N}$ & 12 & 0,78 \\
\hline Tecoma stans (L.) Juss. ex Kunth & Bignoniaceae & Amarelinha & $\mathrm{E}$ & 11 & 0,71 \\
\hline Melia azedarach L. & Meliaceae & Cinamomo & $E$ & 11 & 0,71 \\
\hline Murraya paniculata (L.) Jack & Rutaceae & Falsa Murta & $E$ & 11 & 0,71 \\
\hline $\begin{array}{c}\text { Dypsis decaryi (Jum.) Beentje \& J. } \\
\text { Dransf. }\end{array}$ & Arecaceae & $\begin{array}{l}\text { Palmeira } \\
\text { Triangular }\end{array}$ & $E$ & 10 & 0,65 \\
\hline Roystonea oleracea (Jacq.) O. F. Cook & Arecaceae & Palmeira Imperial & $E$ & 10 & 0,65 \\
\hline Eriobotrya japonica (Thunb.) Lindl. & Rosaceae & Nespera Brasileira & $\mathrm{E}$ & 10 & 0,65 \\
\hline Nerium oleander $\mathrm{L}$. & Apocynaceae & Espirradeira & $\mathrm{E}$ & 9 & 0,58 \\
\hline $\begin{array}{c}\text { Hyophorbe lagenicaulis (L. H. Bailey) } \\
\text { H. E. Moore }\end{array}$ & Arecaceae & Palmeira Garrafa & $E$ & 9 & 0,58 \\
\hline Leucaena leucocephala (Lam.) de Wit & Fabaceae & Leucena & $E$ & 9 & 0,58 \\
\hline Tipuana tipu (Benth.) Kuntze & Fabaceae & Tipuana & $E$ & 9 & 0,58 \\
\hline Eucalyptus sp. & Myrtaceae & Eucalipto & $\mathrm{E}$ & 9 & 0,58 \\
\hline Lithraea molleoides Engl. & Anacardiaceae & Aroeira Brava & $\mathrm{N}$ & 8 & 0,52 \\
\hline Schinus molle L. & Anacardiaceae & Aroeira Salsa & $\mathrm{N}$ & 8 & 0,52 \\
\hline Syzygium jambolanum (Lam.) DC. & Myrtaceae & Jambolão & $E$ & 8 & 0,52 \\
\hline $\begin{array}{c}\text { Handroanthus heptaphyllus (Vell.) } \\
\text { Mattos }\end{array}$ & Bignoniaceae & Ipê Roxo & $\mathrm{N}$ & 7 & 0,45 \\
\hline Carica papaya $L$. & Caricaceae & Mamoeiro & $\mathrm{E}$ & 7 & 0,45 \\
\hline Caesalpinia echinata Lam.1/2E & Fabaceae & Pau Brasil & $\mathrm{N}$ & 7 & 0,45 \\
\hline Cassia fistula $L$. & Fabaceae & Chuva de Ouro & $\mathrm{E}$ & 7 & 0,45 \\
\hline $\begin{array}{c}\text { Senna multijuga (Rich.) H. S. Irwin \& } \\
\text { Barneby }\end{array}$ & Fabaceae & Senna & $E$ & 7 & 0,45 \\
\hline Malpighia glabra L. & Malpighiaceae & Acerola & $\mathrm{E}$ & 7 & 0,45 \\
\hline $\begin{array}{c}\text { Malouetia cestroides (Nees ex Mart.) } \\
\text { Müll. Arg. }{ }^{1}\end{array}$ & Apocynaceae & Peroba-de-leite & $\mathrm{N}$ & 6 & 0,39 \\
\hline Plumeria rubra L. & Apocynaceae & Jasmim Manga & $E$ & 6 & 0,39 \\
\hline $\begin{array}{c}\text { Archontophoenix alexandrae (F. Muell) } \\
\text { H. Wendl. \& Drude }\end{array}$ & Arecaceae & Palmeira-escada & $E$ & 6 & 0,39 \\
\hline $\begin{array}{l}\text { Handroanthus impetiginosus (Mart. ex } \\
\text { DC.) Mattos }\end{array}$ & Bignoniaceae & Pau d'arco & $\mathrm{N}$ & 6 & 0,39 \\
\hline $\begin{array}{c}\text { Chamaecyparis lawsoniana (A. Murray) } \\
\text { Parl. }\end{array}$ & Cupressaceae & Cipreste & $E$ & 6 & 0,39 \\
\hline Aegiphyla sellowiana Cham. & Lamiaceae & Tamanqueira & $\mathrm{N}$ & 6 & 0,39 \\
\hline Cariniana legalis (Mart.) Kuntze ${ }^{1}$ & Lecythidaceae & Jequitiba Rosa & $\mathrm{N}$ & 6 & 0,39 \\
\hline Hibiscus rosa-sinensis $\mathrm{L}$. & Malvaceae & Hibisco & $E$ & 6 & 0,39 \\
\hline Miconia cinnamomifolia (DC.) Naudin ${ }^{1}$ & Melastomataceae & Jacatirão & $\mathrm{N}$ & 6 & 0,39 \\
\hline $\begin{array}{c}\text { Callistemon viminalis (Sol. ex Gaertn.) } \\
\text { G. Don }\end{array}$ & Myrtaceae & Calistemo & $E$ & 6 & 0,39 \\
\hline
\end{tabular}




\begin{tabular}{|c|c|c|c|c|c|}
\hline Hovenia dulcis Thunb. & Rhamnaceae & Uva Japonesa & $E$ & 6 & 0,39 \\
\hline Schefflera actinophylla (Endl.) Harms & Araliaceae & $\begin{array}{l}\text { Árvore-guarda- } \\
\text { chuva }\end{array}$ & $E$ & 5 & 0,32 \\
\hline Cocos nucifera L. & Arecaceae & Coco Anão & $E$ & 5 & 0,32 \\
\hline Gochnatia polymorpha (Less.) Cabrera & Asteraceae & Cambará & $\mathrm{N}$ & 5 & 0,32 \\
\hline Licania tomentosa (Benth.) Fritsch ${ }^{1}$ & Chrysobalanaceae & Oiti & $\mathrm{N}$ & 5 & 0,32 \\
\hline Hymenaea courbaril L. ${ }^{2} \mathrm{LC}$ & Fabaceae & Jatobá & $\mathrm{N}$ & 5 & 0,32 \\
\hline Persea gratissima C. F. Gaertn. & Lauraceae & Abacate Manteiga & $E$ & 5 & 0,32 \\
\hline Tibouchina mutabilis (Vell.) Cogn. ${ }^{1}$ & Melastomataceae & Manacá da Serra & $\mathrm{N}$ & 5 & 0,32 \\
\hline Plinia cauliflora (Mart.) Kausel ${ }^{1}$ & Myrtaceae & Jabuticabeira & $\mathrm{N}$ & 5 & 0,32 \\
\hline Ligustrum lucidum W.T. Aiton & Oleaceae & Ligustro & $E$ & 5 & 0,32 \\
\hline Wodyetia bifurcata A. K. Irvine & Arecaceae & $\begin{array}{l}\text { Palmeira-rabo-de- } \\
\text { raposa }\end{array}$ & $E$ & 4 & 0,26 \\
\hline Dalbergia miscolobium Benth. ${ }^{1}$ & Fabaceae & $\begin{array}{l}\text { Caviúna-do- } \\
\text { cerrado }\end{array}$ & $\mathrm{N}$ & 4 & 0,26 \\
\hline Solanum lycocarpum A. St.-Hil. & Solanaceae & Lobeira & $\mathrm{N}$ & 4 & 0,26 \\
\hline Annona squamosa $\mathrm{L}$. & Annonaceae & Atemóia & $E$ & 3 & 0,19 \\
\hline Caesalpinia pulcherrima (L.) Sw. & Fabaceae & Flamboyanzinho & $E$ & 3 & 0,19 \\
\hline Pachira aquatica Aubl. & Malvaceae & Munguba & $\mathrm{N}$ & 3 & 0,19 \\
\hline Citrus limonia (L.) Osbeck & Rutaceae & Limão Rosa & $\mathrm{E}$ & 3 & 0,19 \\
\hline Liquidambar styraciflua L. & Altingiaceae & Liquidambar & $E$ & 2 & 0,13 \\
\hline Spondias cytherea Sonn. & Anacardiaceae & Cajá-manga & $E$ & 2 & 0,13 \\
\hline Schefflera arboricola (Hayata) Merr. & Araliaceae & Cheflera & $E$ & 2 & 0,13 \\
\hline Araucaria angustifolia (Bertol.) Kuntze & Araucariaceae & Pinheiro do Pará & $\mathrm{N}$ & 2 & 0,13 \\
\hline $\begin{array}{l}\text { Archontophoenix cunninghamii } \mathrm{H} . \\
\text { Wendl. \& Drude }\end{array}$ & Arecaceae & Seafórtia & $E$ & 2 & 0,13 \\
\hline Caryota urens $\mathrm{L}$. & Arecaceae & $\begin{array}{l}\text { Palmeira-rabo-de- } \\
\text { peixe }\end{array}$ & $E$ & 2 & 0,13 \\
\hline $\begin{array}{c}\text { Dypsis lastelliana (Baill.) Beentje \& J. } \\
\text { Dransf. }\end{array}$ & Arecaceae & $\begin{array}{l}\text { Palmeira de } \\
\text { Pescoço Marrom }\end{array}$ & $E$ & 2 & 0,13 \\
\hline Bixa orellana $\mathrm{L}$. & Bixaceae & Urucum & $\mathrm{N}$ & 2 & 0,13 \\
\hline $\begin{array}{l}\text { Euphorbia pulcherrima Willd. ex } \\
\text { Klotzsch }\end{array}$ & Euphorbiaceae & Poinséttia & $E$ & 2 & 0,13 \\
\hline Anadenanthera sp. & Fabaceae & Angico & $\mathrm{N}$ & 2 & 0,13 \\
\hline Bauhinia forficata Link ${ }^{2} \mathrm{LC}$ & Fabaceae & Unha-de-boi & $\mathrm{N}$ & 2 & 0,13 \\
\hline $\begin{array}{c}\text { Centrolobium tomentosum Guillem. ex } \\
\text { Benth. }{ }^{1}\end{array}$ & Fabaceae & Araribá & $\mathrm{N}$ & 2 & 0,13 \\
\hline Platypodium elegans Vogel '2LC & Fabaceae & $\begin{array}{l}\text { Amendoim do } \\
\text { Campo }\end{array}$ & $\mathrm{N}$ & 2 & 0,13 \\
\hline Bertholletia excelsa Bonpl. & Lecythidaceae & Castanheira & $\mathrm{N}$ & 2 & 0,13 \\
\hline Michelia champaca L. & Magnoliaceae & Magnólia Amarela & $E$ & 2 & 0,13 \\
\hline Dimocarpus longan Lour. & Sapindaceae & Olho de Dragão & $E$ & 2 & 0,13 \\
\hline Sambucus canadensis $\mathrm{L}$. & Adoxaceae & Sabugueiro & $\mathrm{E}$ & 1 & 0,06 \\
\hline Tabernaemontana sp. & Apocynaceae & Leiteira & $\mathrm{N}$ & 1 & 0,06 \\
\hline Tabernaemontana catharinensis A. DC. & Apocynaceae & Jasmin & $\mathrm{N}$ & 1 & 0,06 \\
\hline $\begin{array}{c}\text { Dendropanax cuneatus (DC.) Decne. \& } \\
\text { Planch. }\end{array}$ & Araliaceae & Pau-de-tamanco & $\mathrm{N}$ & 1 & 0,06 \\
\hline $\begin{array}{l}\text { Dictyosperma album (Bory) H. L. } \\
\text { Wendl. \& Drude ex Scheff. }\end{array}$ & Arecaceae & Palmeira Princesa & $E$ & 1 & 0,06 \\
\hline Phoenix roebelenii O'Brien & Arecaceae & Tamareira & $E$ & 1 & 0,06 \\
\hline
\end{tabular}




\begin{tabular}{|c|c|c|c|c|c|}
\hline & & Jardim & & & \\
\hline Syagrus oleracea (Mart.) Becc. ${ }^{1}$ & Arecaceae & Guariroba & $\mathrm{N}$ & 1 & 0,06 \\
\hline Enterolobium contortisiliquum (Vell.) & Fabaceae & Timbaúva & $\mathrm{N}$ & 1 & 0,06 \\
\hline \multicolumn{6}{|l|}{ Morong } \\
\hline Machaerium villosum Vogel ${ }^{1}$ & Fabaceae & Jacarandá Paulista & $\mathrm{N}$ & 1 & 0,06 \\
\hline Myroxylon peruiferum L. f. & Fabaceae & Cabreúva & $\mathrm{N}$ & 1 & 0,06 \\
\hline $\begin{array}{c}\text { Stryphnodendron adstringens (Mart.) } \\
\text { Coville }{ }^{1}\end{array}$ & Fabaceae & Barbatimão & N & 1 & 0,06 \\
\hline \multicolumn{5}{|l|}{ Mez } & 0,06 \\
\hline Dombeya wallichii (Lindl.) Baill. & Malvaceae & Astrapéia & $E$ & 1 & 0,06 \\
\hline Guazuma ulmifolia Lam. & Malvaceae & Mutamba & $\mathrm{N}$ & 1 & 0,06 \\
\hline Luehea grandiflora Mart. \& Zucc. & Malvaceae & Açoita-cavalo & $\mathrm{N}$ & 1 & 0,06 \\
\hline Ficus microcarpa L. f. & Moraceae & $\begin{array}{l}\text { Figueira } \\
\text { Lacerdinha }\end{array}$ & $E$ & 1 & 0,06 \\
\hline Ficus catappifolia Kunth \& C.D.Bouché & Moraceae & Figueira & N & 1 & 0,06 \\
\hline Ficus lyrata Warb. & Moraceae & Figueira Violino & $E$ & 1 & 0,06 \\
\hline Eugenia florida DC. ${ }^{1}$ & Myrtaceae & Pitanga-preta & $\mathrm{N}$ & 1 & 0,06 \\
\hline Pimenta dioica (L.) Merr. & Myrtaceae & $\begin{array}{l}\text { Pimenta-da- } \\
\text { jamaica }\end{array}$ & $\mathrm{E}$ & 1 & 0,06 \\
\hline Ouratea spectabilis (Mart.) Engl. ${ }^{1}$ & Ochnaceae & Folha-de-serra & N & 1 & 0,06 \\
\hline Grevillea robusta A. Cunn. ex R. Br. & Proteaceae & Grevilha & $\mathrm{E}$ & 1 & 0,06 \\
\hline Prunus myrtifolia (L.) Urb. & Rosaceae & Pessegueiro-bravo & $\mathrm{N}$ & 1 & 0,06 \\
\hline Prunus persica (L.) Batsch & Rosaceae & Pessegueiro & $E$ & 1 & 0,06 \\
\hline Salix babylonica L. & Salicaceae & Chorão Verdadeiro & $E$ & 1 & 0,06 \\
\hline \multirow[t]{3}{*}{ Solanum pseudoquina A. St.-Hil. } & Solanaceae & Coerana & $N$ & 1 & 0,06 \\
\hline & & Mortas & & 72 & 4,65 \\
\hline & & & Total & 1542 & 100 \\
\hline
\end{tabular}

\section{Análise de Dados}

Conforme os resultados obtidos (Figura 3) pode-se constatar que a maior parte dos indivíduos não apresentaram problemas fitossanitários graves, uma vez que 59,82\% dos indivíduos foram classificados como bons e 32,82\% como regulares (Figura 3A). Quanto à influência do sistema radicular (Figura 3B), a maior parte dos indivíduos (80,56\%) não apresentou conflitos com o contexto em que se inserem por não apresentar suas raízes aparentes no solo. Observou-se, para o diâmetro a altura do peito (Figura 3C), a predominância de indivíduos com DAP "de 5 a menores que $35 \mathrm{~cm}$ " $(80,43 \%)$, já com relação ao diâmetro das copas (Figura 3G), 53,42\% dos indivíduos apresentaram copa "menor que $5 \mathrm{~m}$ ", enquanto $35,21 \%$ apresentam copas com diâmetros "de 5 a menores de $10 \mathrm{~m}$ ". Os resultados obtidos sobre a relação da fiação com as copas (Figura 3F) mostraram que, na maioria dos casos analisados, a fiação elétrica era "inexistente" (56,78\%). 
Figura 3. Frequências observadas para as variáveis relacionadas a A) fitossanidade, B) Influência do sistema radicular no passeio público, C) Diâmetro a altura do peito, D) Altura da primeira bifurcação, E) Altura total, F) Relação entre a fiação elétrica e a copa e G) Diâmetro da copa

Figure 3. Frequencies observed for the variables related to A) Phyto-sanity, B) Influence of root system on the promenade, C) Diameter at breast height, D) Height of first fork, E) Total height, F) Relationship between electrical wiring and the canopy and $\mathrm{G}$ ) Crown diameter
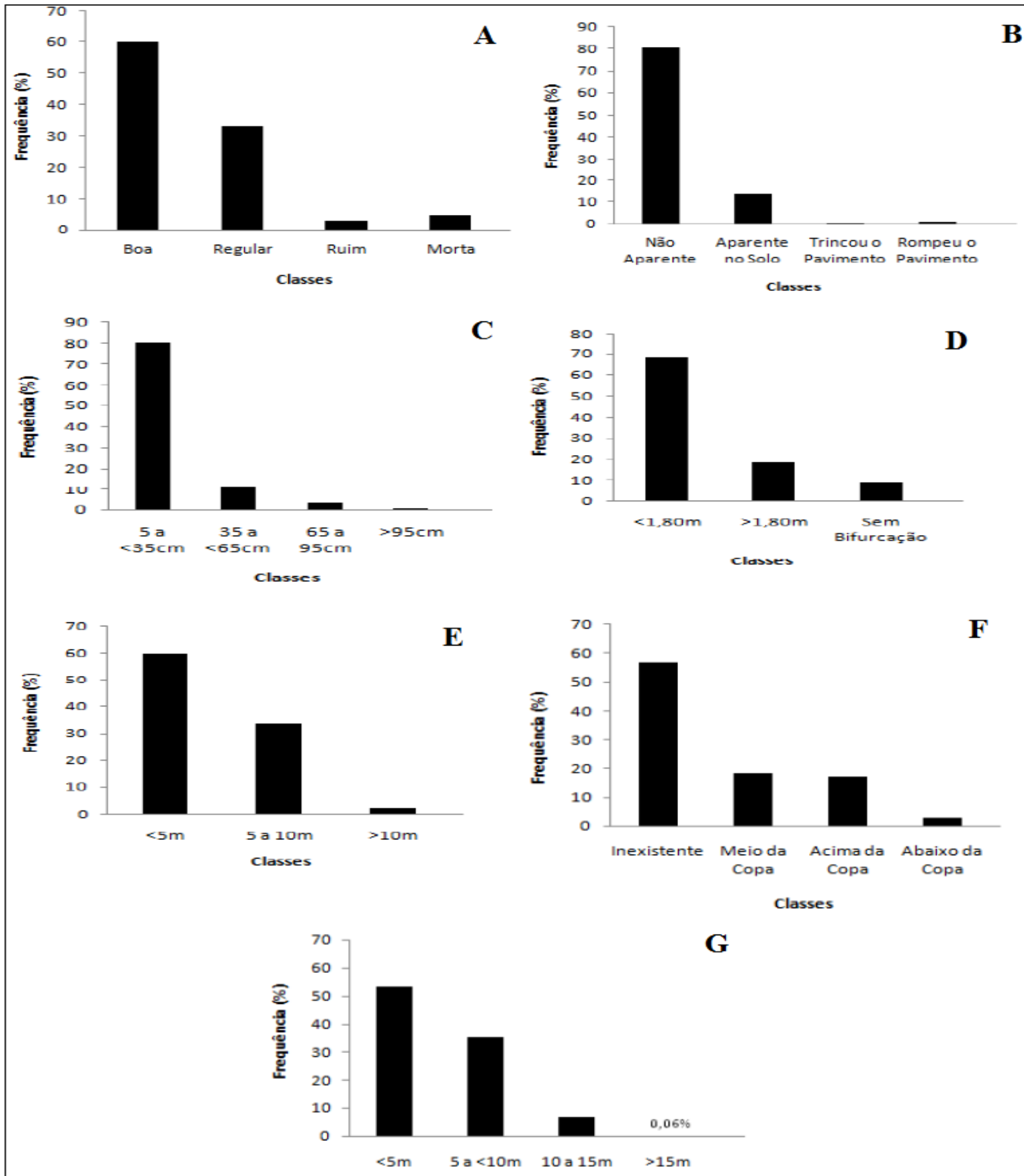

Classes 
Quando analisadas as medidas obtidas para a altura total (Figura 3E), pouco mais da metade dos indivíduos amostrados apresenta-se com "menos de 5m" de altura (59,95\%), e quando verificada a altura da primeira bifurcação (Figura 3D), percebeu-se que em mais da metade dos indivíduos amostrados $(68,22 \%)$ esta ocorre "abaixo de 1,80m".

\section{Análises Gerais}

É recomendado que a composição de espécies se dê da forma mais heterogênea possível, sendo estabelecidos padrões que não excedam $10 \%$ da mesma espécie, $20 \%$ do mesmo gênero e $30 \%$ de uma mesma família botânica para que se mantenha uma grande diversidade de espécies na paisagem urbana (SANTAMOUR-JÚNIOR, 2002). Os resultados obtidos mostram que não existe grande concentração de indivíduos dentro de uma ou poucas espécies, gêneros ou famílias, sendo os padrões encontrados condizentes com os estabelecidos como ideais para que seja melhor controlado o risco de infestações por pragas ou disseminação de doenças entre os indivíduos.

Ainda relacionado à diversidade, tem-se o valor obtido para o Índice de Diversidade de Shannon-Wiener, que se assemelha aos valores encontrados em trabalhos análogos realizados em outros municípios da região, como o realizado por Cardoso-Leite et al. (submetido) que obteve o valor de 3,73 para a cidade de Sorocaba, fornecendo indícios de que a diversidade de espécies se mantém por um certo gradiente regional.

Mesmo diante do valor obtido para diversidade, não é possível afirmar que a escolha das espécies plantadas esteja próxima de um padrão ideal, uma vez que são encontrados muitos indivíduos arbustivos que não maximizam o conforto térmico ao ocuparem o lugar de árvores com copas que forneceriam maior sombreamento do solo, além de serem observadas espécies como Nerium oleander e Spathodea campanulata, as quais não são indicadas para o ambiente urbano devido suas propriedades tóxicas e alucinógenas respectivamente (BATISTA et al., 2013). Além destas, a presença considerável de espécies invasoras como Tecoma stans e Holvenia dulcis pode configurar grande ameaça às espécies nativas locadas em remanescentes vegetais próximos à área urbana, uma vez que a disputa para colonização de novas áreas e competição por recursos e polinizadores/dispersores acaba sendo desigual (SARTORI, 2011).

Diante dos resultados obtidos para fitossanidade, observa-se uma qualidade sanitária satisfatória dos indivíduos amostrados, visto que a grande parte deles foi classificado como bom e regular (Figura 3A). Foi observado, no entanto, que os indivíduos classificados como regulares apresentavam injúrias físicas causadas por entalhes de 
palavras ou inserção de pregos em seus troncos, representando danos causados por ações antrópicas; já aqueles classificados como ruins, apresentavam troncos ocos, com infestações de insetos, um problema também citado em trabalho semelhante realizado por Rossetti et al. (2010), ou severamente queimados devido à cultura do fogo fortemente utilizada na região.

Quanto aos possíveis conflitos do sistema radicular (Figura 3B) com as estruturas urbanas, é perceptível que os resultados estão relacionados com as condições locais, visto que no município há uma baixa porcentagem de ruas com solo impermeabilizado, existindo assim, grande espaço livre permeável ao redor dos indivíduos; dentre as 67 ruas percorridas, apenas 15 eram asfaltadas e destas apenas três ruas apresentavam calçadas impermeabilizadas por pavimento, o que pode facilitar a absorção de água e nutrientes pelas raízes sem que haja necessidade destas ficarem expostas. Mesmo diante deste contexto, ainda foram encontrados indivíduos que apresentaram suas raízes aparentes no solo, tal fato se explica por se tratarem de espécies que possuem como característica a exposição de suas raízes ou de exemplares que apresentam complicações devido à grande compactação do solo. Nas três ruas que apresentam calçamento foi possível diagnosticar 18 indivíduos que se apresentam em conflito com o pavimento urbano, o que pode indicar que o resultado obtido relaciona-se mais com a presença de solo exposto do que com uma possível seleção de espécies com características radiculares compatíveis as exigências do meio urbano.

A predominância de indivíduos com DAP de 5 a menores que $35 \mathrm{~cm}$ (Figura 3C) pode indicar a ocorrência de indivíduos jovens que ainda estão se desenvolvendo, indício que se fortalece ao observar as demais classes de DAP analisadas, uma vez que são encontrados poucos indivíduos nas classes que representavam os maiores valores de DAP, mesmo tendo sido amostrados indivíduos conhecidamente de grande porte. O município de Araçoiaba da Serra encontra-se em constante desenvolvimento, sendo abertas novas ruas e acessos recém-planejados, ocorrendo assim plantios recentes nas novas vias, o que pode explicar a idade jovem dos indivíduos que lá se encontram. Tal fato gera expectativas com relação à arborização em um cenário futuro, pois, segundo Almeida e Rondon Neto (2010), a existência de um alto percentual de indivíduos jovens aumenta a possibilidade de obter-se um número razoável de indivíduos adultos, já que costumam ser relatados altos índices de mortalidade em cenários transitórios.

Diante dos resultados observados para altura da primeira bifurcação (Figura 3D), percebe-se o predomínio de bifurcações que ocorrem abaixo de $1,80 \mathrm{~m}$, resultado semelhante ao encontrado por Benatti et al. (2012), o que pode indicar a utilização de 
indivíduos jovens no plantio realizado a pleno sol sem que houvesse podas de formação no decorrer do seu desenvolvimento para adequação as ruas em que se encontram. Apesar dos fatores ambientais não atuarem isoladamente sobre as plantas, a intensidade luminosa que chega até um indivíduo no decorrer dos seus estádios de crescimento pode ser um fator de modificação na sua conformação final, desta forma, uma das respostas do organismo pode manifestar-se através do desenvolvimento precoce das gemas laterais, ocasionando baixa altura de bifurcação do fuste em organismos plantados a pleno sol (BIONDI; LEAL, 2009). Por conseguinte, observam-se muitas ruas em que as copas das árvores acabam afetando a passagem de carros e pedestres ou que acabam cruzando-se devido ao plantio de indivíduos nos dois lados da via sem que haja alternância de posicionamento entre as mudas, o que, apesar de fornecer sombra e abrigo do sol, pode configurar-se como um problema ao barrar a iluminação pública e sinalização.

Quando analisadas as medidas obtidas para a altura total (Figura 3E), um pouco mais da metade dos indivíduos amostrados apresenta porte baixo, com menos de $5 \mathrm{~m}$ de altura, o que pode indicar a ocorrência de um predomínio de indivíduos jovens no município, uma vez que são encontrados muitos indivíduos com altura abaixo da esperada por pertencerem a espécies de médio e grande porte, como o observado para indivíduos de Jacaranda mimosifolia e Tipuana tipu (LORENZI et al., 2003), sendo então esperado que os mesmos ainda se apresentem em fase de crescimento inicial. Já Araujo et al. (2012), encontraram para este mesmo quesito, resultado oposto, no qual a maior parte dos indivíduos já se apresentava com a altura esperada conforme o porte da espécie a que pertence, tomando-os, assim, como indivíduos adultos.

Os resultados obtidos sobre a relação da fiação com as copas (Figura 3F) mostraram a inexistência da fiação elétrica na maioria dos casos analisados, no entanto, a segunda classe com maior número de indivíduos é também a classe mais conflituosa para esse quesito, na qual a fiação se encontrava no meio da copa. Rodolfo Júnior et al. (2008) encontrou tal situação como sendo a mais expressiva em seu levantamento e a apontou como conflituosa uma vez que tanto podem ocorrer danos às árvores, como choques e queimaduras decorrentes de curtos-circuitos, quanto à distribuição elétrica das redes instaladas já que o contato gerado com as copas pode causar interferências.

Segundo Silva et al. (2007), a existência da fiação elétrica faz com que seja necessário um manejo constante das copas das árvores, além de um melhor planejamento acerca de que espécies podem ser utilizadas em plantios futuros realizados em locais por onde passam os cabos de energia. Indivíduos que apresentam a fiação passando acima de sua copa podem apresentar problemas futuros caso esta venha a atingi-la, sendo 
necessárias podas de segurança ou de adequação como forma preventiva. Já os indivíduos em que a fiação se apresenta abaixo da copa, são necessários cuidados do tipo poda de limpeza ou emergencial para evitar que a queda de galhos possa interferir no fornecimento de energia.

Com relação ao diâmetro das copas (Figura 3G), mais da metade dos indivíduos amostrados apresentaram copa menor que $5 \mathrm{~m}$, configurando contextos adequados ao meio em que se localizam devido à ausência de conflitos com residências e com os demais elementos das vias. No entanto, isso não quer dizer que houve uma seleção de espécies adequadas ao plantio no local, visto que, ao analisar o porte dos indivíduos amostrados, era de se esperar diâmetros de copa maiores, desta forma, atribui-se o resultado obtido a aparente pouca idade da maioria dos indivíduos amostrados e ao manejo destinado, exclusivamente, a copa das árvores que é realizado pela companhia de energia local. Rocha et al. (2004) também percebeu tal relação em seu estudo ao inventariar indivíduos adultos com copas diminutas, alegando que tal fato ocorre uma vez que as podas são efetuadas sempre que os indivíduos apresentavam conflito com a rede elétrica ou postes de iluminação, indicando que essa costuma ser uma prática comum quando se trata do manejo da arborização urbana.

\section{CONCLUSÕES}

A realização deste inventário nos permitiu concluir que existe uma riqueza de espécies relativamente alta, em comparação com outros municípios paulistas, na arborização urbana do município estudado, entretanto a maioria das espécies que a compõe são exóticas. Diante dos dados dendrológicos e os padrões esperados por espécie, é possível perceber que os indivíduos amostrados, em sua maioria, são indivíduos jovens e que apresentam condições satisfatórias de sanidade, entretanto, foram encontrados alguns indivíduos contendo danos decorrentes de pressões antrópicas como injúrias físicas causadas por entalhes de palavras, inserção de pregos em seus troncos e com algumas de suas partes queimadas por conta de incêndios. Devido à falta de seleção de espécies de porte compatível com o local de plantio e a ausência de manejo adequado, foram registrados conflitos com os demais elementos urbanos, como copas se chocando com a fiação elétrica e presença de troncos com baixa bifurcação, o que em muitas ruas, atrapalha a passagem de pedestres e veículos. 
Tendo em vista o nítido crescimento do município, é sabido que ainda haverão mudanças na paisagem e novas adequações ao meio urbano terão de ser feitas com relação a suas estruturas físicas. Eventuais impermeabilizações do solo podem reverter quadros já existentes como o não aparecimento de raízes na superfície do passeio público e a ampliação da rede elétrica pode aumentar os casos de colisão entre as copas e a fiação. Aconselha-se, então, a elaboração de um planejamento pautado em trabalhos relacionados à escolha de espécies adequadas, seguido da elaboração de manuais de manutenção e fiscalização eficazes para as mesmas. Todas estas etapas do planejamento devem ser conhecidas e seguidas por gestores e pela população, à qual devem ser destinadas políticas públicas ligadas à educação ambiental, a fim de que se possa haver sucesso em plantios futuros e maximização dos benefícios gerados pela arborização.

\section{AGRADECIMENTOS}

Os autores agradecem a Secretaria de Agricultura e Meio Ambiente de Araçoiaba da Serra pelo fornecimento de informações e pelo auxílio logístico.

\section{REFERÊNCIAS}

ALMEIDA, D. N.; RONDON NETO, R. M. Análise da arborização urbana de duas cidades da região norte do Estado de Mato Grosso. Revista Árvore, Viçosa - Minas Gerais, v.34, n.5, p.899-906, 2010.

ARAÇOIABA DA SERRA. Plano Municipal de Desenvolvimento Rural Sustentável 2010 - 2013. Prefeitura Municipal de Araçoiaba da Serra, 2010.

ARAÚJO, A. C. B.; GRACIOLI, C. R.; GRIMM E. L.; LONGHI, S. J. Avaliação da florística, do porte e da fitossanidade atual da arborização do parqueinternacional em Sant'ana do Livramento/Riviera, Brasil/Uruguai. Revista da Sociedade Brasileira de Arborização Urbana, Piracicaba - SP, v.7, n.1, p.112-125, 2012.

BATISTA, F. A.; CHAVES, T. P.; FELISMINO, D. de C.; COELHO, I. Inventário qualiquantitativo da arborização urbana na cidade de Remígio, Paraíba. Revista de Biologia e Farmácia - Biofar, Campina Grande - Paraíba, v.9, n.1, p. 70-83, 2013. 
BENATTI, D. P.; TONELLO, K. C.; JÚNIOR, F. C. A.; SILVA, J. M. S. da; OLIVEIRA, I. R. de; ROLIM, E. N.; FERRAZ, D. L. Inventário arbóreo-urbano do município de Salto de Pirapora, SP. Revista Árvore, Viçosa - MG, v.36, n.5, p.887 - 894, 2012.

BIONDI, D.; LEAL, L. Comportamento silvicultural de espécies nativas em viveiro de espera para uso potencial em arborização de ruas. Revista Scientia Forestalis, Piracicaba-SP, v.37, n.83, p.313-319, 2009.

GOMES, M. A. S.; SOARES, B. R. A vegetação nos centros urbanos: considerações sobre os espaços verdes em cidades médias brasileiras. Revista Estudos Geográficos, Rio Claro - São Paulo, v. 1, n. 1, p. 19-29, jun. 2003.

IBGE. Manual técnico da vegetação brasileira, 2012. Disponível em < ftp://geoftp.ibge.gov.br/documentos/recursos_naturais/manuais_tecnicos/manual_tecnico_ve getacao_brasileira.pdf >. Acesso em: 09 abr. 2013.

IBGE. Instituto Brasileiro de Geografia Estatística, 2010. Disponível em: <http://www.ibge.gov.br/home/estatistica/populacao/censo2010/default.shtm> Acesso em: 29 mar. 2013.

IUCN. The IUCN Red List of Threatened species. Disponível em: <http://www.iucnredlist.org/>. Acesso em: 07 mar. 2014.

LORENZI, H. Árvores Brasileiras: Manual de identificação e cultivo de plantas arbóreas nativas do Brasil. 5.ed., v.1, Nova Odessa - SP: Instituto Plantarum, 2008.

LORENZI, H. Árvores Brasileiras: Manual de identificação e cultivo de plantas arbóreas nativas do Brasil. 3.ed., v.2, Nova Odessa - SP: Instituto Plantarum, 2009a.

LORENZI, H. Árvores Brasileiras: Manual de identificação e cultivo de plantas arbóreas nativas do Brasil. 1.ed., v.3, Nova Odessa - SP: Instituto Plantarum, 2009b.

LORENZI, H. Flora Brasileira: Arecaceae (Palmeiras). Nova Odessa - SP: Instituto Plantarum, 2010.

LORENZI, H.; SOUZA, H. M. de; CERQUEIRA, L. S. C. de; COSTA, J. T. de M.; FERREIRA E. Palmeiras brasileiras e exóticas cultivadas. Nova Odessa - SP: Instituto Plantarum, 2004.

LORENZI, H.; SOUZA, S.; TORRES, M. A. V.; BACHER, L. B. Árvores exóticas no Brasil: madeireiras, ornamentais e aromáticas. 2.ed., v.1, Nova Odessa - SP: Instituto Plantarum, 2003. 
LISTA DE ESPÉCIES DA FLORA DO BRASIL, 2013. Disponível em: <http://floradobrasil.jbrj.gov.br/> Acesso em: 15 ago. 2013.

MATIAS, L. F.; CAPORUSSO, D. Áreas verdes urbanas: avaliação e proposta conceitual. In: 1 SIMPÓSIO DE PÓS-GRADUAÇÃO EM GEOGRAFIA DO ESTADO DE SÃO PAULO, 2008, Rio Claro. Anais eletrônicos...Rio Claro: UNESP, 2008. Disponível em: < http://observatoriogeograficoamericalatina.org.mx/egal12/Teoriaymetodo/Metodologicos/10.p df>. Acesso em: 10 jul. 2013.

MEDEIROS, J. de D. Guia de campo: Vegetação do cerrado - 500 espécies. Série Biodiversade 43. Ministério do Meio Ambiente. Brasília, 2011.

RAMOS, V. S.; DURIGAN, G.; FRANCO, G. A. D. C.; SIQUEIRA, M. F. de; RODRIGUES, R. $R$. Árvores da floresta estacional semidecidual - Guia de identificação de espécies. Editora da Universidade de São Paulo: Biota/FAPESP, 2008.

ROCHA, R. T.; LELES, P. S. dos S.; OLIVEIRA-NETO, S. N. de. Arborização de vias públicas em Nova Iguaçu, RJ: o caso dos bairros Rancho Novo e Centro. Revista Árvore, Viçosa - Minas Gerais, v. 28, n. 4, p. 599-607, ago. 2004.

RODOLFO JR., F.; MELO, R. R. de; CUNHA, T. A. da; STANGERLIN, D. M. Análise da arborização urbana em bairros da cidade de Pombal no Estado da Paraíba. Revista da Sociedade Brasileira de Arborização Urbana, Piracicaba - São Paulo, v. 3, n. 4, p. 3-19, out. 2008.

ROSSETTI, A I. N.; TAVARES, A. R.; PELLEGRINO, P. P. Inventário arbóreo em dois bairros paulistanos, Jardim da Saúde e Vila Vera, localizados na subprefeitura de Ipiranga. Revista Árvore, Viçosa - Minas Gerais, v. 34, n. 5, p. 889-898, out. 2010.

SANTAMOUR-JÚNIOR, F. S. Trees for urban planting: diversity unifomuty, and common sense, 2002. Disponível em:< http://www.ces.ncsu.edu/fletcher/programs/nursery/ metria/metria07/m79.pdf> Acesso em: 20 set. 2013.

SARTORI, R. A.; BALDERI, A. P. Inventário da arborização urbana do município de Socorro - SP e proposta de um índice de danos à infra-estrutura das cidades. Revista da Sociedade Brasileira de Arborização Urbana, Piracicaba - São Paulo, v. 6, n. 4, p. 68 89, dez. 2011.

SECRETARIA DO MEIO AMBIENTE DO ESTADO DE SÃO PAULO. Espécies exóticas invasoras: proposta de estratégia para abordar a questão, 2009. Disponível em: < http://www.ambiente.sp.gov.br/consema/files/2011/11/oficio_consema 2009_244/ Especies_Exoticas_Invasoras_propostas_de_estrategia.pdf > Acesso em: 15 ago. $201 \overline{\text {. }}$. 
SHAMS, J. C. A.; GIACOMELI, D. C.; SUCOMINE, N. M. Emprego da arborização na melhoria do conforto térmico nos espaços livres públicos. Revista da Sociedade Brasileira de Arborização Urbana, Piracicaba - São Paulo, v. 4, n. 4, p. 1-16, dez. 2009.

SILVA, A. G. da; PAIVA, H. N. de; GONÇALVES, W. Avaliando a Arborização Urbana. Série Arborização Urbana. 4.ed. Viçosa - MG: Editora Aprenda Fácil, 2007.

TROPICOS Name search - specimens. Missouri Botanical Garden, 2013. Disponível em: <http://www.tropicos.org/Home.aspx>. Acesso em: 02 jul. 2013. 